\title{
Fast Finsler Active Contours and Shape Prior Descriptor
}

\author{
Foued Derraz ${ }^{1,2,4}$, Abdelmalik Taleb-Ahmed ${ }^{2}$, Laurent Peyrodie ${ }^{3}$, \\ Gerard Forzy ${ }^{1,4}$, and Christina Boydev \\ ${ }^{1}$ Faculté Libre de Médecine, 46 Port de Lille, Lille France \\ ${ }^{2}$ LAMIH FRE CNRS 3036, Le Mont Houy, 59313 Valenciennes Université de Valenciennes, \\ Valenciennes, France \\ ${ }^{3}$ HEI - Hautes Etudes d'Ingénieur, Lille, France \\ ${ }^{4}$ Groupe Hospitalier de l'Institut Catholique de Lille, Lille, France \\ foued.derraz@icl-lille.fr, \\ taleb@univ-valenciennes.fr, laurent.peyrodie@hei.fr, \\ gerard.forzy@ghcl. net
}

\begin{abstract}
In this paper we proposed a new segmentation method based Fast Finsler Active Contours (FFAC). The FFAC is formulated in the Total Variation (TV) framework incorporating both region and shape descriptors. In the Finsler metrics, the anisotropic boundary descriptor favorites strong edge locations and suitable directions aligned with dark to bright image gradients. Strong edges are not required everywhere along. We prove the existence of a solution to the new binary Finsler active contours model and we propose a fast and easy algorithm in characteristic function framework. Finally, we show results on some MR challenging images to illustrate accurate.
\end{abstract}

Keywords: Finsler Active contours, Wulff Shape, characteristic function, Shape prior, Primal dual.

\section{Introduction}

The Finsler Active Contours (FAC) has been proposed as natural way for adding directionality to the Active Contours $[1,2,10]$. The utilization of general Finsler metrics instead of Riemannian metrics allows the boundary descriptor to favor appropriate locations and suitable directions [10,12]. The boundary descriptor is weighted by some position and direction-dependent local image information. The local image information can be obtained from a direction-a learned dependent pattern detector. In order to obtain fast and optimal segmentation we proposed both to use local information such anisotropic boundary descriptor [11] and global information such as statistic and geometric shape prior knowledge in the formulation of FAC. The formulation of FAC in Total Variation framework [4,7], allows the convert nonconvex segmentation problem into a convex problem segmentation. The goal is to use an anisotropic boundary descriptor that forces attracting AC at distinct specific positions with particular orientations. Integration position and orientation leads to TV primal dual formulation of segmentation problem. The formulation of the segmentation problem in the characteristic function leads to a fast globally segmentation procedure. We demonstrate the powerful of the proposed segmentation method on some challenging MR images. 


\section{Review of Finsler Active Contours}

In this work, we are interested in a fast segmentation based Finsler Active Contours (FAC) model. We proposed to reformulate traditional FAC in characteristic function framework $(\chi \in[0,1]$ )as combination of finsler, region and shape descriptors in Total variation framework:

$$
\left\{\begin{array}{c}
E(\chi)=\underbrace{\int_{\Omega} k_{f}\left(\mathbf{x}, \mathbf{p}_{\perp}\right)|\nabla \chi(\mathbf{x})| d \mathbf{x}}_{E_{b}(\partial \Omega)} \\
+\lambda_{1} \underbrace{\int_{\Omega} k_{r}(\mathbf{x}, \Omega) \chi(\mathbf{x}) d \mathbf{x}}_{E_{\text {data }}(\mathbf{x}, \Omega)}+\lambda_{2} \underbrace{\int_{\Omega} k_{s}\left(\mathbf{x}, \Omega_{r e f}\right) \chi(\mathbf{x}) d \mathbf{x}}_{E_{\text {shape }}\left(\mathbf{x}, \Omega_{r e f}\right)} \\
\chi=\mathbf{1}_{\left\{\mathbf{x}: \lambda_{1} k_{r}(\mathbf{x}, \Omega)+\lambda_{2} k_{s}\left(\mathbf{x}, \Omega_{r e f}\right)<0 ; \lambda_{1}, \lambda_{2} \in \square^{*+}\right\}}
\end{array}\right.
$$

where $\lambda_{1}, \lambda_{2}$ are the calibration factors, $k_{b}$ is the boundary descriptor, $k_{r}$ is the regions descriptor defined in the same manner as in [9] and $k_{s}$ is the shape prior descriptor defined as in [13] and $\Omega_{\text {ref }}$ the reference shape. In Finsler metrics [10], the traditional isotropic descriptor $k_{b}(\cdot)$ is replaced by an anisotropic Finsler descriptor $k_{f}(\mathbf{x}, \mathbf{p})$ by adding the directionality to the traditional boundary descriptor:

$$
\left\{\begin{array}{c}
k_{f}(\mathbf{x}, \mathbf{p})=k_{b}\left(\mathbf{x}, \mathbf{p}_{\perp}\right) \max \left(\left|\mathbf{p}_{\|}^{1}-\mathbf{p}_{\|}^{2}\right|_{2},\left|\mathbf{p}_{\|}^{1}-\mathbf{p}_{\|}^{2}\right|_{2}\right)^{m=1 o r 2} \\
\mathbf{p}=\left[\mathbf{p}_{\perp}, \mathbf{p}_{\|}\right]
\end{array}\right.
$$

where $\mathbf{p}_{\|}=\left[\mathbf{p}_{\|}^{1}, \mathbf{p}_{\|}^{2}\right]^{\prime}$ denote the tangential direction to the curve $\partial \Omega$ and $\mathbf{p}_{\perp}=\left[\mathbf{p}_{\perp}^{1}, \mathbf{p}_{\perp}^{2}\right]^{\prime}$ denote the normal direction to the curve $\partial \Omega$. Since the desired result is a curve in higher dimensions, a dynamic programming approach is used to determine the implicit convex minimizer of FAC energy [10]. In the next section we are interested in a definition of new convex Finsler descriptor in the TV framework ensuring a globally and optimal segmentation. When other convex or non-convex descriptors such as regions or shape prior descriptors are incorporated within Finsler descriptor, the solution is also implicitly convex.

\section{Finsler Active Contours in the Total Variation Framework}

In this section, we replace the isotropic boundary descriptor in (1) by anisotropic descriptor in TV framework [4, 6, 8, 12]. More formally, let $\left(\psi_{\mathbf{x}}\right)_{\mathbf{x} \in \Omega}$ be a family of weighted anisotropic, positively 1-homogeneous functions, the segmentation problem can be formulated as: 


$$
E_{F A C}(\chi)=\underbrace{\int_{\Omega} \psi_{\mathbf{x}}(\nabla \chi) d \mathbf{x}}_{E_{\text {finster }}()}+\lambda_{1} \underbrace{\int_{\Omega} k_{r}(\mathbf{x}, \Omega) \chi(\mathbf{x}) d \mathbf{x}}_{E_{\text {data }}(\mathbf{x}, \Omega)}+\lambda_{2} \underbrace{\int_{\Omega} k_{s}\left(\mathbf{x}, \Omega_{r e f}\right) \chi(\mathbf{x}) d \mathbf{x}}_{E_{\text {shape }}\left(\mathbf{x}, \Omega_{\text {ref }}\right)}
$$

Where $\psi$ is induced by potential shapes noted as Wulff shapes $W_{\psi}[6,8,11]$.

\section{Definition}

Let $\psi: \square^{2} \rightarrow \square \quad$ be $\quad$ a convex, positively 1-homogeneous function $\psi(\lambda \nabla \chi)=\lambda \psi(\nabla \chi)$, for $\lambda>0$.

The Wulff shape $W_{\psi}$ convex, bounded and closed set $\left(0 \in W_{\psi}\right)$ is defined as:

$$
\begin{aligned}
& W_{\psi}=\left\{\forall \mathbf{x} \in \Omega \subset \square^{2}, \nabla \chi \in \square^{2}:\langle\mathbf{p}, \nabla \chi\rangle \leq \psi(\nabla \chi)\right\} \\
& \text { such } W_{\psi}=\left\{-\mathbf{p}: \mathbf{p} \in W_{\psi}\right\} \\
& \text { and } \psi(\nabla \chi)=\max _{\mathbf{p} \in-W_{\psi}}(\langle\mathbf{p}, \nabla \chi\rangle)=\max _{\mathbf{p} \in W_{\psi}}(\langle-\mathbf{p}, \nabla \chi\rangle)
\end{aligned}
$$

where $\langle$,$\rangle denote the inner product in \square^{2}$.

The nature of solution of (3) was already shown for the TV [4, 6, 11] by rewriting the anisotropic TV in terms of characteristic function and extended to general families Wulff shape.

\section{Lemma}

Let $\psi$ be a positively 1 -homogeneous function, and the descriptors $k_{r}$ and $k_{s}$. Then any global minimizer of (3) can be considered as a global minimize.

Proof: Assume $\chi^{*}: \Omega \rightarrow[0,1]$ is a global minimizer of (3). The corresponding characteristic function $\hat{\chi}$ is then defined as:

$$
\hat{\chi}(\mathbf{x})= \begin{cases}1 & \text { if } \chi^{*}(\mathbf{x}) \geq 0 \\ 0 & \text { if } \chi^{*}(\mathbf{x})<0\end{cases}
$$

If $\nabla \hat{\chi} \neq 0$ has the same direction as $\nabla \chi^{*}$, then we can write $\nabla \hat{\chi}=c \nabla \chi^{*}, c \in \square^{*_{+}}$. The dual energy of (3) is given by:

$$
E_{F A C}(\mathbf{p})=\int_{\Omega} \min \left(0, \operatorname{div}(\mathbf{p})+\lambda_{1} k_{r}(\mathbf{x}, \Omega)+\lambda_{2} k_{s}\left(\mathbf{x}, \Omega_{r e f}\right)\right) d \mathbf{x}
$$

which is maximized with respect to a vector field $\mathbf{p}$ subject to $-\mathbf{p} \in W_{\psi_{x}}$. By inserting the respective constraints on $\chi$ and pusing the dirac function $\delta$, the primal (3) and dual (6) energies can be stated as:

$$
E^{*}(\chi)=E_{F A C}(\chi)+\int_{\Omega} \delta_{[0,1]}(\chi(\mathbf{x})) d \mathbf{x}
$$


and we can expressed the dual energy as:

$$
E_{F A C}^{*}(\mathbf{p})=E_{F A C}(\mathbf{p})-\int_{\Omega} \delta_{W_{\psi}}(-\mathbf{p}) d \mathbf{x}
$$

We use the Karush-Kuhn-Tucker (KKT) conditions to prove the optimality of $\hat{\chi}[3]$. Let $\mathbf{p}^{*}$ be the corresponding dual solution for $\chi^{*}$. The KKT conditions stated as:

$$
\nabla \chi^{*} \in \partial \int \delta_{w_{\psi}}\left(-\mathbf{p}^{*}\right)-\operatorname{div}\left(\mathbf{p}^{*}\right) \in \lambda_{1} \partial \int k_{r}(\mathbf{x}, \Omega) \chi^{*} d \mathbf{x}+\lambda_{2} \partial \int k_{s}\left(\mathbf{x}, \Omega_{r e f}\right) \chi^{*} d \mathbf{x}+\delta_{[0,1]}\left(\chi^{*}\right)
$$

Hence the KKT conditions can be applied point-wise. Therefore, $\left(\chi^{*}, \mathbf{p}^{*}\right)$ are minimizers of the primal energy (7) and its corresponding dual energy (8) if and only if:

$$
\nabla \chi^{*} \in \delta_{W_{\psi}}\left(-\mathbf{p}^{*}\right)-\operatorname{div}\left(\mathbf{p}^{*}\right) \in \lambda_{1} k_{r}(\mathbf{x}) \chi^{*}+\lambda_{2} k_{s}\left(\mathbf{x}, \Omega_{r e f}\right) \chi^{*}+\delta_{[0,1]}\left(\chi^{*}\right)
$$

Then, we established that $\nabla \hat{\chi}$ is sub gradient of $\delta_{w_{\psi}}\left(-\mathbf{p}^{*}\right)$ :

$$
\delta_{W_{\psi}}\left(-\mathbf{p}^{*}\right)+(\nabla \hat{\chi})^{T}\left(\mathbf{p}-\mathbf{p}^{*}\right) \leq \delta_{w_{\psi}}(-\mathbf{p})
$$

Since $-\mathbf{p}^{*} \in W_{\psi}$ and $-\mathbf{p} \in W_{\psi} \Rightarrow \delta_{W \psi}\left(-\mathbf{p}^{*}\right)=0$ and $\delta_{W \psi}(-\mathbf{p})=0$ and we can write:

$$
(\nabla \hat{\chi})^{T}\left(\mathbf{p}-\mathbf{p}^{*}\right)=c\left(\nabla \chi^{*}\right)^{T}\left(\mathbf{p}-\mathbf{p}^{*}\right) \leq 0, c \in \square^{+}
$$

We can establish that $\nabla \hat{\chi} \in \delta_{W_{\psi}}\left(-\mathbf{p}^{*}\right)$.

To establish that $-\operatorname{div}\left(\mathbf{p}^{*}\right)$ is sub gradient [3] in (12), we consider two cases. In the first case $\chi^{*}$ is already either 0 or 1 , then $\hat{\chi}=\chi^{*}$. The second case $\chi^{*}$ is in the open interval $] 0,1\left[\right.$, then $\partial \delta_{[0,1]}\left(\chi^{*}\right)=0$, since $\delta_{[0,1]}(\cdot)$ is constant in $[0,1]$ :

$$
\delta_{[0,1]}(\hat{\chi}) \leq \delta_{[0,1]}(\chi)+\operatorname{div}\left(\mathbf{p}^{*}\right)(\chi-\hat{\chi})
$$

Then $-\operatorname{div}\left(\mathbf{p}^{*}\right) \in \lambda_{1} k_{r}(\mathbf{x}, \Omega) \hat{\chi}+\lambda_{2} k_{s}\left(\mathbf{x}, \Omega_{r e f}\right) \hat{\chi}+\delta_{[0,1]}(\hat{\chi})$, thus $\left(\hat{\chi}, \mathbf{p}^{*}\right)$ also satisfies the KKT conditions and $\hat{\chi}$ is therefore a global binary minimizer.

\section{Finsler Active Contours in the TV Framework}

In this section, we consider that $\psi(\nabla \chi)=\max _{\mathbf{p} \in W_{\psi}}\left(-\mathbf{p}^{T} \nabla \chi\right)$, the energy in (7) and (8) can be formulated in a primal-dual setting as: 


$$
E(\chi, \mathbf{p})=\int_{\Omega} \underbrace{\max _{-\mathbf{p} \in W_{\psi}}\langle\mathbf{p}, \nabla \chi(\mathbf{x})\rangle}_{\psi_{\mathbf{x}}(\chi)}\rangle \mathbf{x}+\int_{\Omega}\left\{\begin{array}{l}
\lambda_{1} k_{r}(\mathbf{x}, \Omega) \\
+\lambda_{2} k_{s}\left(\mathbf{x}, \Omega_{r e f}\right)
\end{array}\right\} \chi(\mathbf{x}) d \mathbf{x}
$$

and the respective gradient descent (for $\chi$ ) and ascent (for $\mathbf{p}$ ) equations are:

$$
\left\{\begin{array}{c}
\frac{\partial \chi(\mathbf{x}, \tau)}{\partial \tau}=\operatorname{div}(\mathbf{p})-\lambda_{1} k_{r}(\mathbf{x}, \Omega)-\lambda_{2} k_{s}\left(\mathbf{x}, \Omega_{r e f}\right), \chi(., \tau=0)=\mathbf{1}_{\min \left(\operatorname{dist}\left(\mathbf{x}, \partial \Omega_{0}\right)\right)} \\
\frac{\partial \mathbf{p}}{\partial \tau}=-\nabla \chi(\mathbf{x}, \tau), \quad \mathbf{p}_{0}=\text { design asin } \sec t 5.2
\end{array}\right.
$$

where $\tau$ is an artificial time parameter, $\partial \Omega_{0}$ is the initial contour curve. Enforcing the constraints on $\chi$ and $\mathbf{p}$ is both done by clamping $[0,1]$, and reprojecting $\mathbf{p}$ onto the feasible set $W_{\psi_{\mathbf{x}}}$.

The Energy based region term is usually defined as a domain integral of the region descriptor $k_{r}$ :

$$
E_{\text {data }}(I, \Omega)=\int_{\Omega} \sqrt{p(I \mid \Omega) p\left(I \mid \Omega \backslash \Omega_{I}\right)} d \mathbf{x}
$$

Where the region descriptor based on Bhattachryya distance [9] is calculated using the gradient shape tool [5]:

$$
k_{r}(\mathbf{x}, \Omega)=\frac{1}{2}\left\{\begin{array}{l}
\sqrt{p_{\text {in }}(I(\mathbf{x}), \Omega) p_{\text {out }}(I, \Omega)}\left(\frac{1}{\left|\Omega_{\text {in }}\right|}-\frac{1}{\left|\Omega_{\text {out }}\right|}\right)+ \\
\int_{R^{+}} \frac{1}{\left|\Omega_{\text {out }}\right|} \sqrt{\frac{p_{\text {in }}(I, \Omega)}{p_{\text {ref }}(I, \Omega)}} \cdot\left(G_{\sigma_{\mathrm{ker}}}(I-I(\Omega))-\sqrt{p_{\text {out }}(I, \Omega)}\right) d I \\
-\int_{R^{+}} \frac{1}{\left|\Omega_{\text {in }}\right|} \sqrt{\frac{p_{\text {out }}(I, \Omega)}{p_{\text {in }}(I, \Omega)}} \cdot\left(G_{\sigma_{\mathrm{ker}}}(I-I(\Omega))-\sqrt{p_{\text {in }}(I, \Omega)}\right) d I
\end{array}\right\}
$$

We therefore estimate density by Parzen kernel, which can better describe the regions. This method estimates the probability density function based on the histograms, using a smoothed Gaussian kernel:

$$
p\left(I, \Omega_{i}\right)=\frac{1}{\left|\Omega_{i}\right|} \int_{\Omega_{i}} G_{\sigma}\left(I-I\left(\Omega_{i}\right)\right) d \mathbf{x}
$$

where $G_{\sigma}$ denote the Gaussian kernel and $\sigma^{2}$ the variance.

The shape prior descriptor is defined as the Euclidean distance between the evolving Legendre moment region $\eta(\Omega)$ and the reference shapes $\left\{\chi_{\text {ref }}^{i}, i=1, \ldots, N\right\}$ :

$$
E_{\text {shape }}\left(\mathbf{x}, \Omega_{\text {ref }}\right)=\int_{\Omega} k_{\text {shape }}\left(\mathbf{x}, \chi_{\text {ref }}^{i}\right) d \mathbf{x}=\sum_{p, q}^{p+q \leq N}\left|\eta_{p q}(\chi)-\eta_{p q}\left(\chi_{\text {ref }}^{i}\right)\right|^{2}
$$


where the $\eta_{p q}$ are defined as follows, using the geometric moments $M_{p q}$ and the coefficients $a_{p q}$ of the Legendre polynomials [13]:

$$
\eta_{p q}=\frac{(2 p+1)(2 q+1)}{4} \sum_{u=0}^{p} \sum_{v=0}^{q} a_{p u} a_{q v} M_{u v}
$$

Where $M_{p q}(\chi)=\frac{1}{\chi^{\frac{p+q+2}{2}}} \int_{\Omega}(x-\bar{x})^{p}(y-\bar{y})^{q} d x d y$ and $(\bar{x}, \bar{y})$ are the shape barycenter coordinates. In the next section we established our based fast FAC segmentation method.

\section{Results}

\subsection{Data and Protocol}

In this section, we provide prostate segmentation results for two data sets obtained from Saint Philibert Hospital Lille France. The MR images are pre-processed through the following pipeline: 1) spatial registration, 2) noise removal and 3) intensity standardization. We use the T1 weighted and T2 weighted MR sequences. The image sizes are $256 \times 256$ pixels, each slice thickness is $3.5 \mathrm{~mm}$ with spacing between slices of $3.9 \mathrm{~mm}$.

\subsection{Finsler Shape Design}

There is a wide spread of the possibilities to design $\psi_{\mathbf{x}}$. In Fig. 1(a), the Wulff shape is composed by a half-circle with radius 1 and a circular segment combining gradient direction with gradient magnitude. Designing $\psi_{\mathrm{x}}$ in such manner allows straightforward reprojection for the vector field after the gradient ascent update (15).

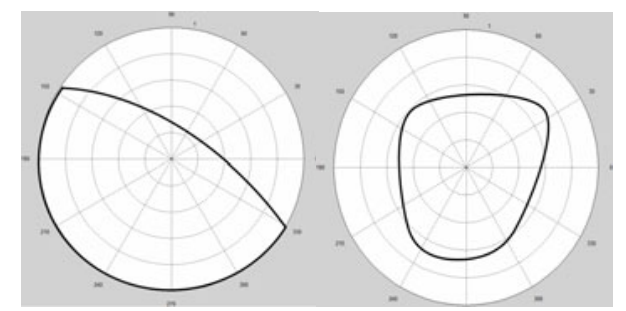

(a)

(b)

a) Wullf Shape for TV FAC Wullf b) Shape for Traditional FAC

Fig. 1. Construction of Wulff shape for Finsler Active Contours

\subsection{Learned Shapes}

The prostate learned shapes are designed by an expert using manual segmentation. In the figure 2, the object shape is segmented using 20 learned shapes and the segmentation is done using statistical and geometric shape prior. 

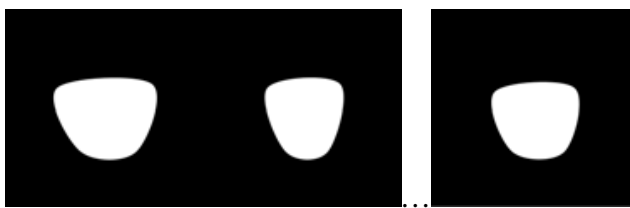

$$
\chi_{\text {ref }}^{1}, \chi_{\text {ref }}^{2}, \ldots, \chi_{\text {ref }}^{20}
$$

Fig. 2. Construction of Wulff shape for Finsler Active Contours

In the next section we introduced our interactive user term used to suitability segmentation method.

\subsection{Segmentation Results}

To quantify the accuracy of the segmentation, we measured the Dice Similarity Coefficient (DSC) between the manually segmented prostate and our segmentation method. We provide not only qualitative results (Fig. 3), but also give quantitative results in the form of the DSC to illustrate the viability of the proposed method in the context of prostate segmentation (see Table. 1).

Table 1. Quantitative evaluation of the segmentation

\begin{tabular}{|l|c|c|}
\hline Patient no & DSC of FAC & DSC of FAC TV \\
\hline Patient no 01 & $80,02 \%$ & $85,63 \%$ \\
\hline Patient no 02 & $79,1 \%$ & $82,69 \%$ \\
\hline Patient no 03 & $75,06 \%$ & $79,23 \%$ \\
\hline Patient no 04 & $77,21 \%$ & $79,75 \%$ \\
\hline Patient no 05 & $75,7 \%$ & $79,30 \%$ \\
\hline
\end{tabular}

To quantify the accuracy of the segmentation, we measured the overlap between the segmented prostate areas defined by manual segmentation and our segmentation method.

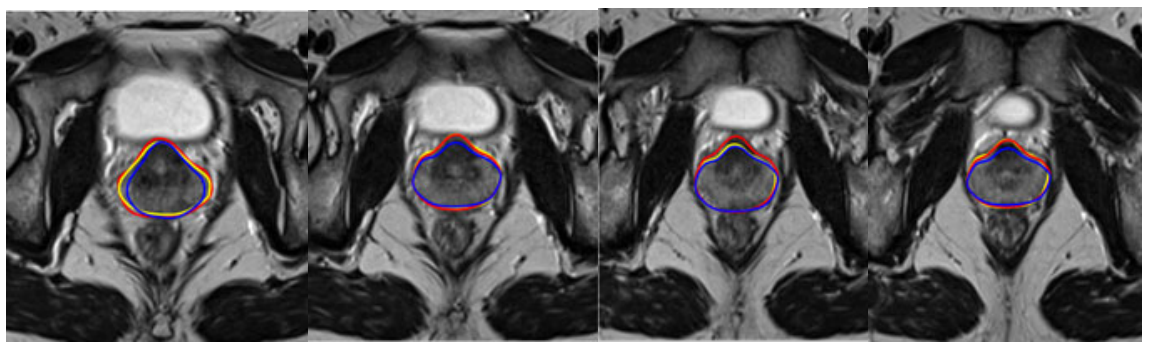

Fig. 3. Segmentation by TV FAC. In blue color traditional FAC, red color segmentation results of our method and in yellow color manual segmentation. 


\section{Conclusion}

We developed a fast globally segmentation based convex Finsler active contours model for binary segmentation in TV framework incorporating statistical and shape prior knowledge. The position and orientation are dependent on prior for the boundary segmentation in Finsler metrics. Finsler active contours provide an alternative approach to integrating image-based priors on the location and orientation of the traditional boundary descriptor. Future work will address extending other classes of energies [9] that can be optimized in TV framework.

\section{References}

1. Chan, T.F., Vese, L.: Active contours without edges. IEEE Trans. IP 10(2), 266-277 (2001)

2. Appleton, B., Talbot, H.: Globally minimal surfaces by continuous maximal flows. IEEE Trans. PAMI 28(1), 106-118 (2006)

3. Borwein, J.M., Lewis, A.S.: Convex analysis and nonlinear optimization: theory and examples, 2nd edn. Canadian Mathematical Society (2000)

4. Bresson, X., Esedoglu, S., Vandergheynst, P., Thiran, J., Osher, S.: Fast Global Minimization of the Active Contour/Snake Model. JMIV 28(2) (2007)

5. Herbulot, A., Besson, S.J., Duffiner, S., Barlaud, M., Aubert, G.: Segmentation of vectorial image features using shape gradients and information measures. JMIV 25(3), 365-386 (2006)

6. Caselles, V., Chambolle, A.: Anisotropic curvature-driven flow of convex sets. Nonlinear Analysis 65(8), 1547-1577 (2006)

7. Rousson, M., Paragios, N.: Prior Knowledge, Level Set Representations and Visual Grouping. IJCV 76(3), 231-243 (2008)

8. Peng, D., Osher, S., Merriman, B., Zhao, H.: The Geometry of Wulff Crystal Shapes and Its Relations with Riemann Problems. In: Nonlinear PDE' 1998, pp. 251-303 (1998)

9. Michailovich, O., Rathi, Y., Tannenbaum, A.: Image Segmentation Using Active Contours Driven by the Bhattacharyya Gradient Flow. IEEE Trans. IP 16(11), 2787-2801 (2007)

10. Melonakos, J., Pichon, E., Angenent, S., Tannenbaum, A.: Finsler active contours. IEEE Trans. PAMI 30(3), 412-423 (2008)

11. Chan, T.F., Esedoglu, S.: Aspects of total variation regularized L1 function approximation. SIAM JAM 65(5), 1817-1837 (2005)

12. Chern, S., Shen, Z.: Riemann-Finsler Geometry. World Scientific (2005)

13. Foulonneau, A., Charbonnier, P., Heitz, F.: Affine-Invariant Geometric Shape Priors for Region-Based Active Contours. IEEE Trans. PAMI 28(8), 1352-1357 (2006)

14. Zhang, X., Burger, M., Osher, S.: A Unified Primal-Dual Algorithm Framework Based on Bregman Iteration. J. Sci. Comput. 46(1), 20-46 (2010)

15. Lellmann, J., Breitenreicher, D., Schnörr, C.: Fast and Exact Primal-Dual Iterations for Variational Problems in Computer Vision. In: Daniilidis, K., Maragos, P., Paragios, N. (eds.) ECCV 2010, Part II. LNCS, vol. 6312, pp. 494-505. Springer, Heidelberg (2010) 